\title{
Possible Causes of Discordance in Refraction in Monozygotic Twins: Nearwork, Time Outdoors and Stochastic Variation
}

\author{
Xiaohu Ding, ${ }^{1}$ Yin Hu, ${ }^{1}$ Xinxing Guo, ${ }^{1,2}$ Xiaobo Guo, ${ }^{3}$ Ian Morgan, ${ }^{1,4}$ and Mingguang He ${ }^{1,5}$ \\ ${ }^{1}$ State Key Laboratory of Ophthalmology, Zhongshan Ophthalmic Center, Sun Yat-sen University, Guangzhou, China \\ ${ }^{2}$ Dana Center of Preventive Ophthalmology, Wilmer Eye Institute, Johns Hopkins University, Baltimore, Maryland, United States \\ ${ }^{3}$ Department of Statistics, School of Mathematics \& Computational Science, Sun Yat-Sen University, Guangzhou, China \\ ${ }^{4}$ Research School of Biology, College of Medicine, Biology and Environment, Australia National University, Canberra, Australia \\ ${ }^{5}$ Centre for Eye Research Australia, Ophthalmology, Department of Surgery, University of Melbourne, Melbourne, Australia
}

Correspondence: Mingguang He, State Key Laboratory of Ophthalmology, Zhongshan Ophthalmic Center, Sun Yat-sen University, \#54 Xianlie Road, Yuexiu District, Guangzhou, Guangdong 510060, People's Republic of China; mingguang_he@yahoo.com.

Submitted: April 19, 2018 Accepted: October 8, 2018

Citation: Ding X, Hu Y, Guo X, Guo X, Morgan I, He M. Possible causes of discordance in refraction in monozygotic twins: nearwork, time outdoors and stochastic variation. Invest $O p h$ thalmol Vis Sci. 2018;59:5349-5354. https://doi.org/10.1167/iovs.18-24526
Punpose. To evaluate the impact of differences in nearwork and time spent outdoors on difference in refraction in monozygotic (MZ) twins.

Methods. Data on MZ twins aged 7 to 18 years from the Guangzhou Twin Eye Study were used in this analysis. A standard questionnaire was administered by personal interview to estimate time spent on nearwork and time spent outdoors. Spherical equivalent (SE) was measured by autorefraction under cycloplegia. The interaction between age and nearwork or time spent outdoors was also estimated.

Results. A total of $490 \mathrm{MZ}$ twin pairs (233 male and 257 female) were eligible in this analysis, the mean age was $13.14 \pm 2.49$. In the mixed-effects model, nearwork difference was a risk factor of discordance in myopic SE $(\beta=-0.11$ diopter $(\mathrm{D}) / \mathrm{h}, P=0.009)$, the overall association between time outdoors difference and SE discordance was not significant $(\beta=$ $-0.89(\mathrm{D}) / \mathrm{h}, P=0.120)$ although an interaction between time spent outdoors difference and age was detected $(\beta=0.07(\mathrm{D}) / \mathrm{h}, P=0.002)$. Furthermore, difference in nearwork and time outdoors explained about $1.8 \%$ and $2.5 \%$ of the variation in SE discordance, respectively.

Concuusions. Given the very marked genetic similarity of MZ twins, and the small effects of known risk factors on SE discordance, we suggest that the SE discordance across MZ twins largely results from stochastic variations at the genomic or epigenetic levels, or from uncollected environmental factors.

Keywords: monozygotic twin, nearwork, outdoor activity, myopia, stochastic variation
$M$ yopia is one of the most common eye disorders worldwide. ${ }^{1}$ The prevalence is particularly high in East Asia. $^{2-5}$ While simple myopia (myopia less than -6 Diopters $[\mathrm{D}])$ is usually correctable and has a low risk of blinding complications, high myopia (myopia at least $-6 \mathrm{D}$ ) is associated with increased risk of complications that potentially lead to irreversible visual impairment, such as retinal detachment, myopic macular degeneration, staphyloma, and glaucoma. ${ }^{6}$ Therefore, myopia is becoming a critically important public health problem worldwide.

There has been a long debate about the relative roles of variation at the genomic levels and in environmental exposures in the etiology of myopia. The high heritability of refraction observed in twin studies is consistent with the hypothesis that genetic factors play a role ${ }^{7-9}$ although this is not conclusive because the heritability values found in broader family and population-wide studies are considerably lower. ${ }^{10-12}$ The greater risk of developing myopia in children who have myopic parents compared to those with nonmyopic parents is also consistent with a role of genetic factors, although this effect could be interpreted as that myopic parents create more myopigenic environments for their children. ${ }^{13,14}$ Populationbased genome-wide association studies (GWAS) have identified about 150 significant loci for myopia, but altogether they explain less than $8 \%$ of the variation ${ }^{15}$ In parallel, environmental factors also seem to play a significant role. In particular, there is considerable evidence that people with higher educational achievements are more likely to be myopic and highly myopic - an effect which could be, at least in part, due to axial elongation associated with prolonged near work. ${ }^{16}$ In addition, recent work has shown that the amount of time spent outdoors can be a protective environmental factor. ${ }^{17-19}$ One hypothesis is that this may be due to the inhibition of axial elongation by the increased release of retinal dopamine in response to the greater light exposures experienced outdoors during daylight hours. ${ }^{20,21}$ Overall, it is now generally accepted that there are a number of relatively rare forms of clearly genetic myopia, but that only impacts of environmental factors can explain the rapid increases in the prevalence of myopia that have taken place in the countries with well-developed education systems in East and Southeast Asia.

However, previous epidemiological studies were unable to disentangle the influence of variations in genetic background from variations in environmental exposure. The discordance of traits within MZ twin pairs offers a unique opportunity to study environmental effects because the pair-wise genetic back- 
ground is much more similar than that of randomly compared individuals, even if there is some variation between "identical" twins. $^{22}$

In the current investigation, we therefore used an MZ cotwin control methodology, which provides twin-pairs that are matched for genetic background, sex, and age, and matched as possible for genetic background and shared family environment. This matching allows us to examine how much nearwork and time spent outdoors influence the within-pair discordance in spherical equivalent (SE) while largely eliminating confounding genetic effects. We also examined the development of discordance in refraction between the two eyes in one individual, which is conceptually a similar problem to that seen with discordance between MZ twins, namely how do different phenotypic outcomes arise from very similar, if not identical, genotypes. Overall, we have found very limited, albeit significant, evidence for a role for environmental factors in the development of between-pair discordance.

\section{MeTHODS}

\section{Participants}

Participants of this study were recruited from the Guangzhou Twin Eye Study, which is population-based study and has been described elsewhere. ${ }^{23,24}$ Briefly, all twins were identified by the Resident Registry and then verified by both door-to-door visits and mailouts. The demographic characteristics of the identified twins were similar to the general population in Guangzhou as a whole. The study was launched in 2006 and has now completed more than ten consecutive annual followup examinations. More than 1200 pairs of eligible twins aged 7 to 15 years were enrolled at baseline. Cycloplegic refraction was performed at baseline and follow-up visits. In the present study, data collected in the visit in 2009 was used for statistical analyses. Those with manifest strabismus, amblyopia, nystagmus, postrefractive surgery, or any ocular disease resulting in best-corrected visual acuity less than 20/25 were excluded. The study was conducted in accordance with the tenets of the Declaration of Helsinki and was approved by the Ethics Review Board of the Zhongshan Ophthalmic Center of Sun Yat-Sen University. Written informed consent was obtained from parents or legal guardians of the participants. Zygosity of all same-sex twin pairs was determined using the 16-multiplex short-tandem repeat (STR) DNA typing system (Gene Print Power Plex, Promega Corporation, Madison, WI, USA). ${ }^{25}$ Opposite-sex twin pairs were deemed to be dizygotic.

\section{Eye Examinations and Refraction Measurements}

The eye examinations began with an uncorrected visual acuity test at $4 \mathrm{~m}$ using a retroilluminated $\log$ MAR chart with tumbling-E optotypes (Precision Vision, La Salle, IL, USA). Ocular motility was evaluated with cover tests conducted at 0.5 and 4.0 meters. Corneal light reflex was used to quantify the degree of tropia according to the Hirschberg method. The light reflex at the pupillary margin is about $2 \mathrm{~mm}$, in the midiris region is about $4 \mathrm{~mm}$, and in the limbus is about $6 \mathrm{~mm}$ from the pupillary center (with a 4-mm pupil), which corresponds to $15^{\circ}, 30^{\circ}$, and $45^{\circ}$ of deviation. Axial length (AL) was measured by optical biometry using a commercial device (IOLMaster; Carl Zeiss Meditec, Jena, Germany).

Cycloplegia was induced with 2 drops of 1\% cyclopentolate, administered 5 minutes apart, and a third drop administered 20 minutes later. Cycloplegia and pupillary dilation were evaluated after an additional 15 minutes. Cycloplegia was considered complete if the pupil was dilated to $6 \mathrm{~mm}$ or greater and the pupillary light reflex was absent. If not, an additional 20 minutes of observation was allowed, and refractive measurements were taken regardless of the presence or absence of light reflex. Refraction was performed using an auto-refractor (Topcon KR-8800, Tokyo, Japan) after cycloplegia.

\section{Questionnaire}

The questionnaire used in this study was designed by a World Health Organization (WHO) working group (Supplementary Material S1). It included questions on indoor and outdoor activities on weekdays and weekends separately. In each section, daily activity was divided into four types: nearwork activity (including reading, writing, and drawing), middledistance activity (including watching television or movies and playing video games), indoor leisure activity (including singing, housework, and dancing indoors), and time spent outdoors (including sports and walking outside). Participants were asked to report daily time spent on each of the activities: using categories of not at all, less than one hour, or more than one hour. If "more than one hour" was reported, the exact time spent on the activity was further specified. The questionnaire was administered by an in-person interview conducted by a single trained interviewer who was fluent in both Mandarin and Cantonese, the two most spoken languages in Guangzhou.

\section{Data Analysis}

Spherical equivalent (SE) was calculated as spherical power plus half cylindrical power. Data of the right eyes were used for $\mathrm{MZ}$ discordance analysis because the refractive errors in the right and left eyes were highly correlated $(r=0.92, P<0.001$, Pearson correlation analysis). Furthermore, in order to explore the effect of gene and environment on the variation of intereye SE, we explored the heritability of inter-eye SE difference as continuous traits or binary trait on anisometropia. The definition of anisometropia is the intereye SE difference greater than $2 \mathrm{D}$.

On weekdays, the amount of time for each vision-related activities was defined as the sum of time spent in class and before/after class, according to our previous study. ${ }^{26}$ Based on typical pattern of behaviors in school, we calculated the amount of nearwork during school classes as follows: nearwork time $=$ total class time $\times 0.4 ;$ middle-distance work $=$ total class time $\times 0.4$; indoor leisure time $=$ total class time $\times 0.1$ and outdoor time $=$ total class time $\times 0.1$. Outside of school hours, the time spent on the four types of visual activity was reported directly in responses to the questionnaires.

During school terms, the average time for each type of activities was calculated as $(5 \times$ weekday $+2 \times$ weekend $) / 7$. During holidays, the pattern of visual activities was assumed to be that seen during weekends. In China, there are 9 months of semester days (from February to July and from September to December) and 3 months of summer/winter holidays each year. The average nearwork and time spent outdoors per day in the past year was calculated as $(9 \times$ semester day time $+3 \times$ holiday time)/12.

The SE discordance was defined as the SE of older twin minus the SE of younger twin, based on the order of delivery in the birth certificate. The discordance on environmental parameters were defined in the same manner. A linear mixedeffects model was used to account the correlation between MZ twin pairs, in which the random effect term was used to account for the correlation between MZ twin pairs, and the fixed effect terms was used to model the effects of nearwork and time outdoors. The variation in SE discordance between MZ twin pairs was decomposed into variations in difference in 
TABLE 1. The Demography of Monozygotic Twin Pairs $(N=980$ Individual)

\begin{tabular}{lccccr}
\hline & $\boldsymbol{N}$ & Spherical Equivalent, D & Age, $\mathbf{y}$ & Nearwork/Day, h & Time Spent Outdoors/Day, h \\
\hline Male & 466 & $-1.27 \pm 2.08$ & $12.92 \pm 2.08$ & $3.94 \pm 1.27$ & $1.40 \pm 0.84$ \\
Female & 514 & $-1.71 \pm 2.18$ & $13.36 \pm 2.49$ & $4.50 \pm 1.54$ & $1.33 \pm 0.87$ \\
Total & 980 & $-1.50 \pm 2.14$ & $13.14 \pm 2.49$ & $4.24 \pm 1.45$ & $1.36 \pm 0.86$
\end{tabular}

nearwork between MZ twin pairs, differences in time spent outdoors between MZ twin pairs and the unique environmental effects. A $P$ value of less than 0.05 was considered to be statistically significant. The statistical analyses were performed using SAS statistical software (SAS Institute, Inc., Cary, NC, USA). Full details of the linear mixed-effects model are given in the online (Supplementary Material S2).

\section{RESULTS}

In a total of $521 \mathrm{MZ}$ twin pairs examined, 31 pairs were excluded because of incomplete questionnaire responses, incomplete cycloplegia or tropia; the remaining 490 pairs of MZ twins (233 male and 257 female) were included in this analysis. The mean age was $13.1 \pm 2.5$ years (range, 7 to 18 ) in 2009. The characteristics of the MZ twins included in the analysis were shown in Table 1 . A high correlation between SE of the right and left eyes was found (Pearson, $r=0.92, P<$ 0.001), data from right eyes were chosen for calculating SE discordance within MZ twin pairs. The distribution of pairwise SE discordance in MZ pairs and intereye SE difference between the two eyes in MZ singletons are shown in the Figure. The standard deviation of intereye SE variation in MZ singletons was significantly greater than the SE discordance in MZ pairs (1.13 D vs. $0.81 \mathrm{D}, P<0.0001$, SD test)

In the mixed-effects model analysis of SE discordance, older age $(\beta=-0.34, P<0.001)$ and greater nearwork difference $(\beta$ $=-0.23, P<0.001$ ) were risk factors for SE discordance, (Table $2)$. Interaction between age and difference in time spent outdoors was significant for SE discordance $(\beta=0.07, P=$ 0.002 ), while difference in time spent outdoors itself did not have a significant effect $(\beta=-0.89, P=0.120)$. The across-twin- pair analyses showed that variation in difference in nearwork and difference in time spent outdoors between the MZ twins explained about $1.8 \%$ and $2.5 \%$ of the total SE discordance, respectively.

We wanted to put some limits on how much of the total variation could be caused by stochastic variation. We assumed that the difference between two eyes in one individual is most likely to come from stochastic variation (or from measurement error), since the two eyes generally share the same genes and environmental exposures. In the Figure, we can see that the stochastic variation explained about $2 / 3$ of the within-MZ twin variance.

Furthermore, since $\mathrm{AL}$ is the most important ocular biometry determining SE, we also explored the effect of nearwork and time outdoors difference on AL discordance in the same way. Interaction between age and environment was not statistically significant and was not included in the final model. Table 3 shows that greater positive nearwork difference was a risk factor for longer $\mathrm{AL}(\beta=0.05, P=0.001)$, while time spent outdoors was not a protective factor. The variation in nearwork difference explained about $1.9 \%$ of total AL variation.

The heritability of intereye SE variation, as well as the heritability of anisometropia, were also evaluated using MZ and DZ twins. The intraclass correlation was calculated, but it was only about 0.02 in both MZ and DZ twin pairs, so it was not suitable for heritability estimation with continuous variables. The concordance of anisometropia was $92.7 \%$ in $\mathrm{MZ}$ twins and $91.7 \%$ in DZ twins, which was not significantly different between the two types of twins. All the above indicated that there was no significant genetic effect on the intereye SE variation.

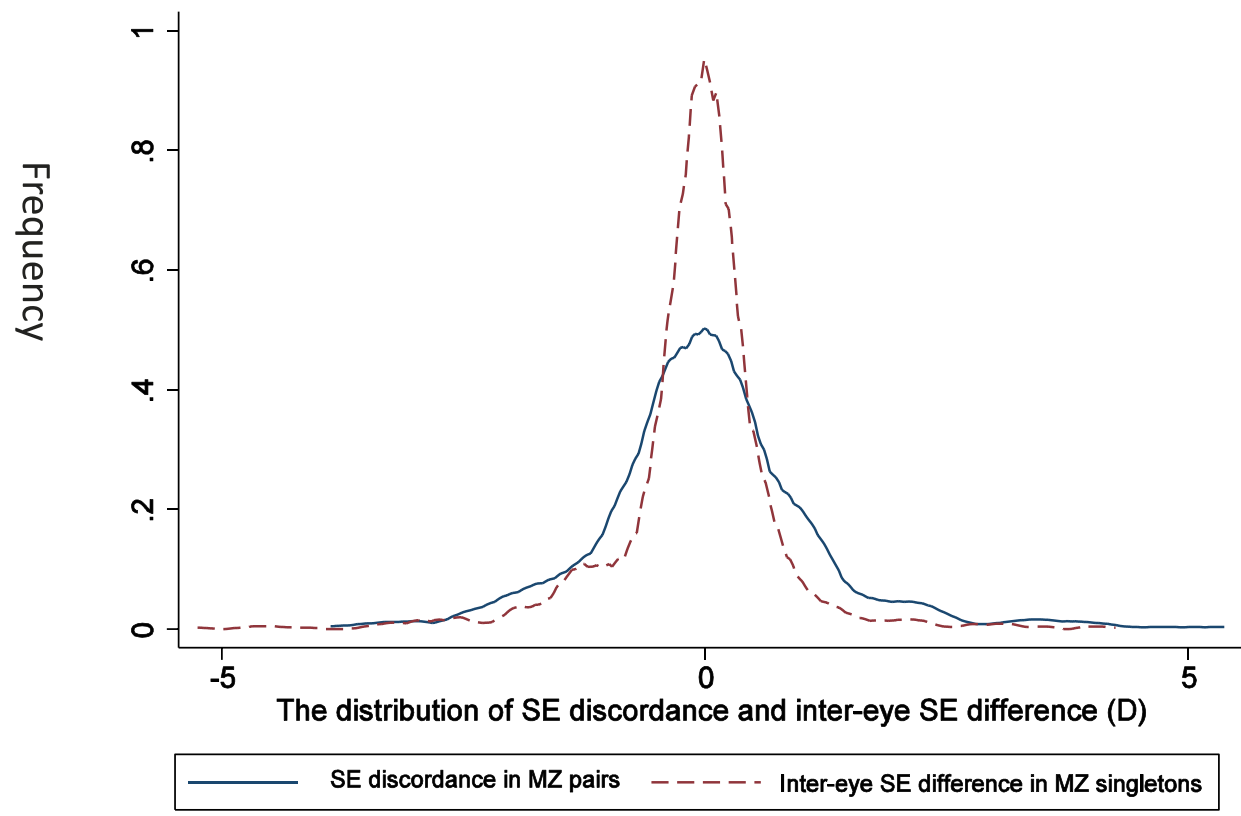

Figure. The distribution of SE discordance within MZ twin pair and SE difference between two eyes in single person. 
TABle 2. Linear Mixed Model for Discordance in SE in MZ Twins

\begin{tabular}{lrr}
\hline \multicolumn{1}{c}{ Fixed Effect } & Coefficient & 95\% CI \\
\hline Age, D/y & -0.34 & -0.50 to -0.18 \\
Sex, female vs. male & -0.31 & -0.68 to 0.07 \\
Nearwork difference, D/h* & -0.001 \\
Time outdoors difference, D/h* & -0.23 & -0.67 to 0.22 \\
Nearwork difference $\times$ age* & -0.89 & -1.50 to -0.27 \\
Time spent outdoors difference $\times$ age* & 0.01 & -0.02 to 0.04 \\
\end{tabular}

Bold values represent results that are significant at the $P<0.05$ level. 95\% CI, 95\% Confidence interval.

$*$ Difference in nearwork and difference in time spent outdoors is defined as $1.8 \%$ and $2.5 \%$ of the variation in discordance in spherical equivalent, respectively.

\section{Discussion}

We used cotwin methodology in our study to explore the role of discordance in difference in nearwork and difference in time spent outdoors on discordance in SE after controlling for genetic predisposition. By using a mixed-effects model, we found that age and nearwork were risk factors for discordance in SE. An interaction effect between time outdoors difference and age was detected, suggesting the association between outdoor time difference and SE discordance was mainly driven by their effects in older age group children, perhaps in the older group, the twins and their cotwins are old enough to behave differently.

This MZ cotwin discordance methodology potentially provides researchers with valuable insight into the contribution of environmental factors, based on the assumption that genetic background is very similar, if not identical, in MZ twins. In this scenario, MZ phenotypic discordance between MZ twins might be explained by nonshared environmental effects, such as epigenetic variation, stochastic variation or measurement error. The cotwin discordance method has been attempted in a few studies that involve some complex diseases, such as multiple sclerosis. ${ }^{27,28}$ Ramessur et al. ${ }^{29}$ have explored the discordance among MZ twins having myopia and found that more myopic twins have higher occupational status, resident in urban area, performing more nearwork than their twin. Previous studies investigated the MZ discordance in disease outcomes dichotomously. In the present study, we explored discordance as a continuous parameter.

In our study, a questionnaire designed by a WHO working group was adopted and was conducted by in-person interview. Both cross-sectional and longitudinal epidemiologic studies have shown that time spent outdoors is a protective factor against the development of myopia in European and Chinese populations. ${ }^{17-19,30}$ In our cotwin cross-sectional study, we further confirm the protective role of time spent outdoors in MZ twin pairs.

In a population-based cross-sectional study, potential nongenetic risk factors, including amount of near work, age, and sex, together with IQ, which may have a significant genetic input, explained $10 \%$ to $12 \%$ of total variation in SE. Unfortunately, the importance of time outdoors had not been recognized at that time, and thus data on this parameter were not included. ${ }^{31}$ In our analysis, the collected environmental factors suggested by previous evidence (namely nearwork and outdoor time) only accounted for $3.5 \%$ of the total phenotypic variation. The questionnaire estimates are known to be imprecise, and hence it is possible that these estimates may have failed to capture the true variance of nearwork and time spent outdoors. An alternative explanation is that other important environmental factors were not included in our data collection, although at present, the two examined, nearwork and time outdoors, appear to be the best documented. Moreover, the measurement error and the potentially nonlinear exposure-outcome relationship may have caused the low degree of variance explained by the two environmental factors. Since the MZ twins tend to share very similar environmental factors, the conclusions on the contribution of nearwork and outdoor time may not be directly applicable to general population.

Stochastic variation may also explain part of the phenotypic variance among $M Z$ twin pairs. In order to examine the effect of stochastic variation, we plotted the distribution of intereye $\mathrm{SE}$ variation in the same individual assuming this intereye variation is largely due to random variation at genome or epigenome level. We estimated the heritability of anisometropia (intereye SE difference $>2 \mathrm{D}$ ) and intereye variation in SE. This approached zero. The intereye variance $(\mathrm{SD}=0.81 \mathrm{D})$ was about $2 / 3$ of the variance of phenotypic discordance $(\mathrm{SD}=$ $1.13 \mathrm{D})$. This finding suggests that stochastic variance did explain major parts of the pairwise discordance in MZ pairs.

In this population-based twin study, the twins were recruited independent of zygosity and eye disease status, and the sample is likely to be representative of the population of those ages Consistent with this idea, the distribution of refraction in our twin cohort was comparable to an agematched sample derived from general population. ${ }^{2}$

However, our findings should be interpreted in light of some limitations. First, MZ twin pairs have similar, but not identical, genetic background. Postzygotic effects, could introduce differences. ${ }^{32,33}$ Secondly, the myopia-related environmental data were collected by a questionnaire in a retrospective fashion, which may lead to some recall bias.

TABLE 3. Linear Mixed Model for Discordance in AL in MZ Twins

\begin{tabular}{lccr}
\hline \multicolumn{1}{c}{ Fixed Effect } & Coefficient & 95\% CI & $\boldsymbol{P}$ Value \\
\hline Age, D/y & 0.12 & 0.07 to 0.16 & $<\mathbf{0 . 0 0 1}$ \\
Sex, female to male & -0.51 & -0.70 to -0.31 & $<1$ \\
Difference in nearwork, D/h & 0.05 & 0.02 to 0.08 & $\mathbf{0 . 0 0 1}$ \\
Difference in time spent outdoors, D/h & -0.02 & -0.06 to 0.30 & 0.001 \\
& & & 0.410
\end{tabular}

Nearwork difference and difference in time spent outdoors explained $1.9 \%$ and $0.1 \%$ of the variation in AL discordance, respectively. Bold values represent results that are significant at the $P<0.05$ level. 
Objective measurement of these environmental risk factors is therefore important.

We cannot clearly distinguish between measurement errors and stochastic variations in this analysis. But it is possible to set a limit to their impact on variation, by looking at the correlations between eyes in refraction. The correlation coefficient in these studies is 0.92 , and in many other studies ranges from 0.8 up. Assuming that the departures from prefect correlation are due to these two factors, has an important practical consequence. GWAS studies aiming to explain the variation in refraction in terms of identified SNPs, as well as the role of environmental factors cannot expect to reach $100 \%$ power. Assuming around $20 \%$ of the variance as associated with stochastic variation and measurement errors, this would lower the target power from $100 \%$ to $80 \%$, which would reduce the level of "missing heritability" but by no means eliminate it.

In conclusion, our results confirm that nearwork is a risk factor of myopia, while time spent outdoors confers protective effects among more time outdoors with elder age, based on an analysis on differences in environmental exposures and their association with discordance in refraction. We found that differences in both nearwork and time spent outdoors accounted for less than $3.0 \%$ of the variation in refraction discordance. Given the very marked genetic similarity between MZ twins and the relatively small effects of known environmental risk factors, we suggest that the discordance in refraction between MZ twins may largely result from unknown or uncollected environmental factors, despite the limited evidence for factors other than time outdoors and factors associated with education such as near work, or stochastic variations at the genomic or epigenetic levels. In the case of the difference between eyes, it is possible that essentially stochastic somatic mosaicism could also play a role. ${ }^{34,35}$

\section{Acknowledgments}

Supported by the National Natural Science Foundation of China (81125007) and Fundamental Research Funds of the State Key Laboratory, the University of Melbourne Research Accelerator Program and the CERA Foundation (MH). The Centre for Eye Research Australia receives Operational Infrastructure Support from the Victorian State Government. The authors alone are responsible for the content and writing of the paper.

Disclosure: X. Ding, None; Y. Hu, None; X. Guo, None; X. Guo, None; I. Morgan, None; M. He, None

\section{References}

1. Morgan IG, French AN, Ashby RS, et al. The epidemics of myopia: aetiology and prevention. Prog Retin Eye Res. 2018; 62:134-149.

2. Hur YM, Zheng Y, Huang W, et al. Comparisons of refractive errors between twins and singletons in Chinese school-age samples. Twin Res Hum Genet. 2009;12:86-92.

3. Jung SK, Lee JH, Kakizaki H, Jee D. Prevalence of myopia and its association with body stature and educational level in 19year-old male conscripts in seoul, South Korea. Invest Ophthalmol Vis Sci. 2012;53:5579-5583.

4. He M, Zeng J, Liu Y, et al. Refractive error and visual impairment in urban children in southern china. Invest Ophthalmol Vis Sci. 2004;45:793-799.

5. Lee YY, Lo CT, Sheu SJ, Lin JL. What factors are associated with myopia in young adults? A survey study in Taiwan military conscripts. Invest Ophthalmol Vis Sci. 2013;54: 1026-1033.
6. Saw SM, Gazzard G, Shih-Yen EC, Chua WH. Myopia and associated pathological complications. Ophthalmic Physiol Opt. 2005;25:381-391.

7. Hammond CJ, Snieder H, Gilbert CE, Spector TD. Genes and environment in refractive error: the twin eye study. Invest Ophthalmol Vis Sci. 2001;42:1232-1236.

8. Lyhne N, Sjolie AK, Kyvik KO, Green A. The importance of genes and environment for ocular refraction and its determiners: a population based study among $20-45$ year old twins. Br J Ophthalmol. 2001;85:1470-1476.

9. Dirani M, Chamberlain M, Shekar SN, et al. Heritability of refractive error and ocular biometrics: the Genes in Myopia (GEM) twin study. Invest Ophthalmol Vis Sci. 2006;47:47564761 .

10. Chen CY, Scurrah KJ, Stankovich J, et al. Heritability and shared environment estimates for myopia and associated ocular biometric traits: the Genes in Myopia (GEM) family study. Hum Genet. 2007;121:511-520.

11. Klein AP, Suktitipat B, Duggal P, et al. Heritability analysis of spherical equivalent, axial length, corneal curvature, and anterior chamber depth in the Beaver Dam Eye Study. Arch Ophthalmol. 2009;127:649-655.

12. Vitart V, Bencic G, Hayward C, et al. Heritabilities of ocular biometrical traits in two croatian isolates with extended pedigrees. Invest Ophthalmol Vis Sci. 2010;51:737-743.

13. Ip JM, Huynh SC, Robaei D, et al. Ethnic differences in the impact of parental myopia: findings from a population-based study of 12-year-old Australian children. Invest Ophthalmol Vis Sci. 2007; 48:2520-2528.

14. Xiang F, He M, Morgan IG. The impact of parental myopia on myopia in Chinese children: population-based evidence. Optom Vis Sci. 2012;89:1487-1496.

15. Tedja MS, Wojciechowski R, Hysi PG, et al. Genome-wide association meta-analysis highlights light-induced signaling as a driver for refractive error. Nat Genet. 2018;50:834-848.

16. Katz J, Tielsch JM, Sommer A. Prevalence and risk factors for refractive errors in an adult inner city population. Invest Ophthalmol Vis Sci. 1997;38:334-3340.

17. Rose KA, Morgan IG, Ip J, et al. Outdoor activity reduces the prevalence of myopia in children. Ophthalmology. 2008;115: 1279-1285.

18. Dirani M, Tong L, Gazzard G, et al. Outdoor activity and myopia in Singapore teenage children. Br J Ophthalmol. 2009;93:997-1000.

19. Wu PC, Tsai CL, Hu CH, Yang YH. Effects of outdoor activities on myopia among rural school children in Taiwan. Ophthalmic Epidemiol. 2010;17:338-42.

20. Gao Q, Liu Q, Ma P, Zhong X, Wu J, Ge J. Effects of direct intravitreal dopamine injections on the development of lidsuture induced myopia in rabbits. Graefes Arch Clin Exp Ophthalmol. 2006;244:1329-1335.

21. McCarthy CS, Megaw P, Devadas M, Morgan IG. Dopaminergic agents affect the ability of brief periods of normal vision to prevent form-deprivation myopia. Exp Eye Res. 2007;84:100107.

22. Boomsma D, Busjahn A, Peltonen L. Classical twin studies and beyond. Nat Rev Genet. 2002;3:872-882.

23. He M, Ge J, Zheng Y, et al. The Guangzhou Twin Project. Twin Res Hum Genet. 2006;9:753-757.

24. Zheng Y, Ding X, Chen Y, He M. The Guangzhou Twin Project: an update. Twin Res Hum Genet. 2013;16:73-78.

25. Moretti TR, Baumstark AL, Defenbaugh DA, Keys KM, Smerick JB, Budowle B. Validation of short tandem repeats (STRs) for forensic usage: performance testing of fluorescent multiplex STR systems and analysis of authentic and simulated forensic samples. J Forensic Sci. 2001;46:647-660. 
26. He M, Xiang F, Zeng Y, et al. Effect of time spent outdoors at school on the development of myopia among children in china: a randomized clinical trial. JAMA. 2015;314:11421148.

27. Biegstraaten M, van Schaik IN, Aerts JM, et al. A monozygotic twin pair with highly discordant Gaucher phenotypes. Blood Cells Mol Dis. 2011;46:39-41.

28. Baranzini SE, Mudge J, van Velkinburgh JC, et al. Genome, epigenome and RNA sequences of monozygotic twins discordant for multiple sclerosis. Nature. 2010;464:13511356.

29. Ramessur R, Williams KM, Hammond CJ. Risk factors for myopia in a discordant monozygotic twin study. Ophthalmic Physiol Opt. 2015;35:643-651.

30. Jones-Jordan LA, Sinnott LT, Cotter SA, et al. Time outdoors, visual activity, and myopia progression in juvenile-onset myopes. Invest Ophthalmol Vis Sci. 2012;53:7169-7175.
31. Saw SM, Tan SB, Fung D, et al. IQ and the association with myopia in children. Invest Ophthalmol Vis Sci. 2004;45: 2943-2498.

32. Raj A, van Oudenaarden A. Nature, nurture, or chance: stochastic gene expression and its consequences. Cell. 2008; 135:216-226.

33. Jimenez-Gomez JM, Corwin JA, Joseph B, et al. Genomic analysis of QTLs and genes altering natural variation in stochastic noise. PLoS Genet. 2011;7:e1002295.

34. McConnell MJ, Moran JV, Abyzov A, et al. Intersection of diverse neuronal genomes and neuropsychiatric disease: The Brain Somatic Mosaicism Network. Science. 2017;356: eaal1641.

35. Forsberg LA, Gisselsson D, Dumanski JP. Mosaicism in health and disease - clones picking up speed. Nat Rev Genet. 2017; 18:128-142. 